\title{
BMJ Open Trends and socioeconomic disparities in all-cause mortality among adults with diagnosed diabetes by race/ethnicity: a population-based cohort study - USA, 1997-2015
}

Carla Mercado (D) , ${ }^{1}$ Gloria Beckles, ${ }^{1}$ Yiling Cheng, ${ }^{1}$ Kai McKeever Bullard, ${ }^{1}$ Sharon Saydah, ${ }^{1}$ Edward Gregg, ${ }^{2}$ Giuseppina Imperatore ${ }^{1}$

To cite: Mercado C, Beckles G, Cheng Y, et al. Trends and socioeconomic disparities in all-cause mortality among adults with diagnosed diabetes by race/ethnicity: a population-based cohort study - USA, 1997-2015. BMJ Open 2021;11:e044158. doi:10.1136/ bmjopen-2020-044158

- Prepublication history and additional supplemental material for this paper are available online. To view these files, please visit the journal online (http://dx.doi.org/10.1136/ bmjopen-2020-044158).

Received 25 August 2020 Revised 27 February 2021 Accepted 15 March 2021

Check for updates

(C) Author(s) (or their employer(s)) 2021. Re-use permitted under CC BY-NC. No commercial re-use. See rights and permissions. Published by BMJ.

${ }^{1}$ Division of Diabetes Translation, Centers for Disease Control and

Prevention, Atlanta, Georgia, USA

${ }^{2}$ Imperial College London,

London, UK

Correspondence to

Dr Carla Mercado;

cmercado@cdc.gov

\section{ABSTRACT}

Objectives By race/ethnicity and socioeconomic position (SEP), to estimate and examine changes over time in (1) mortality rate, (2) mortality disparities and (3) excess mortality risk attributed to diagnosed diabetes (DM).

Design Population-based cohort study using National Health Interview Survey data linked to mortality status from the National Death Index from survey year up to 31 December 2015 with 5 years person-time.

Participants US adults aged $\geq 25$ years with $(31586)$ and without (332 451) DM.

Primary outcome Age-adjusted all-cause mortality rate for US adults with DM in each subgroup of SEP (education attainment and income-to-poverty ratio (IPR)) and time (1997-2001, 2002-2006 and 2007-2011).

Results Among adults with DM, mortality rates fell from $23.5 / 1000$ person-years $(p-y)$ in 1997-2001 to $18.1 / 1000$ p-y in 2007-2011 with changes of $-5.2 / 1000$ p-y for nonHispanic whites; $-5.2 / 1000$ p-y for non-Hispanic blacks; and $-5.4 / 1000$ p-y for Hispanics. Rates significantly declined within SEP groups, measured as education attainment $(<$ high school=-5.7/1000 p-y; high school graduate $=-4.2 / 1000 \mathrm{p}-\mathrm{y}$; and $>$ high school $=-4.8 / 1000$ $\mathrm{p}-\mathrm{y}$ ) and IPR group (poor $=-7.9 / 1000 \mathrm{p}-\mathrm{y}$; middle income $=-4.7 / 1000 \mathrm{p}-\mathrm{y}$; and high income $=-6.2 / 1000$ $\mathrm{p}$-y; but not for near poor). For adults with DM, statistically significant all-cause mortality disparity showed greater mortality rates for the lowest than the highest SEP level (education attainment and IPR) in each time period. However, patterns in mortality trends and disparity varied by race/ethnicity. The excess mortality risk attributed to DM significantly decreased from 1997-2001 to 20072011, within SEP levels, and among Hispanics and nonHispanic whites; but no statistically significant changes among non-Hispanic blacks.

Conclusions There were substantial improvements in all-cause mortality among US adults. However, we observed SEP disparities in mortality across race/ethnic groups or for adults with and without DM despite targeted efforts to improve access and quality of care among disproportionately affected populations.

\section{Strengths and limitations of this study}

- A population-based study on multidimensional association and disparities between socioeconomic position (SEP), race/ethnicity, diabetes and mortality along with whether any changes have occurred since 1997.

- A series of consecutive national representative surveys (1997-2011) were linked to latest available mortality data through 31 December 2015.

- Aside from investigating changes in mortality rates over time, this study measured the mortality disparity from lower to higher SEP rankings and how those disparities have changed over time.

- Since diabetes and SEP statuses were self-reported and only measured at baseline, misreporting and status changes during the course of the follow-up period may have occurred.

\section{INTRODUCTION}

From mid-1990s, diabetes mellitus has increased rapidly in the USA with an estimated 23.1 million people had diagnosed diabetes, $9.4 \%$ of the total population, in 2015. ${ }^{1}$ Prevalence and incidence of diagnosed diabetes affects racial/ethnic groups disproportionately, with greater and plateauing estimates among non-Hispanic blacks and Hispanics than the lower declining ones for non-Hispanic whites. ${ }^{2-4}$ Additionally, diabetes prevalence is greater at lower socioeconomic position (SEP) levels, measured by education attainment and income, than higher levels. ${ }^{5}$ Although several national, state and local programmes and initiatives were developed to reduce diabetes and eliminate diabetes-associated disparities, ${ }^{7}$ marked racial/ethnic and socioeconomic disparities in prevalence of diabetes were reported between 2004 and 2010 with increased 
socioeconomic disparities magnitude among adults with diagnosed diabetes over time..$^{8-10}$

Diabetes socioeconomic patterning is associated with reduced access to care, poor quality of care, underuse of preventive health measures and healthcare behaviours that provide pathways to increased mortality risk. ${ }^{11}$ SEP (measured by either education attainment, wealth, income and/or income-to-poverty ratio (IPR)) has been reported to be inversely associated with all-cause mortality risk. ${ }^{12-16}$ When the magnitudes of absolute educational disparities (slope index of inequality, SII) were assessed, adults with diabetes experienced a greater all-cause mortality burden associated with low levels of education than those without diabetes. ${ }^{12-16}$ Additionally, the educational gradient in allcause mortality rates was present in non-Hispanic white and non-Hispanic blacks, but not among Hispanics. ${ }^{12-16}$

The socioeconomic mortality association has typically been examined separately without examining the intersectionality of known related characteristics, such as race/ethnicity or diabetes. This approach ignores that individuals inhabit multiple social statuses simultaneously, that these statuses interact to shape the health risk patterns experienced, and thereby the health disparities observed. ${ }^{16}$ Investigation in population-level race/ethnicspecific and SEP-specific mortality rate changes among adults with diagnosed diabetes could inform national, state and local efforts aimed at reducing diabetes-related disparities. Furthermore, examining changes in excess mortality risk attributed to diabetes could determine if diabetes-related disparity has narrowed. This study aims to examine whether and to what extent race/ethnic: (1) SEP-specific mortality rates have changed among US adult population during 1997-2011 with diagnosed diabetes, (2) SEP disparities in all-cause mortality exist and changed from 1997 to 2011 among adults with diagnosed diabetes and (3) has the excess mortality risk attributed to diabetes (rate difference between those with and without diabetes) in SEP-specific groups changed during 1997-2011.

\section{METHODS}

\section{Data and population}

Data from the National Health Interview Surveys (NHIS) for the years 1997-2011 were linked with death certificates from the National Death Index (NDI) to obtain the most current mortality status through 31 December 2015. ${ }^{17}$ NHIS is an annual ongoing cross-sectional household interview survey (about 35000 households per year) of a nationally representative civilian, non-institutionalised sample conducted by the Centers for Disease Control and Prevention (CDC)'s National Center for Health Statistics (NCHS) ${ }^{18} 19$ Participation in NHIS is voluntary and confidentiality is assured under the Public Health Service Act Section 308(d). NHIS data from 1997 through 2011 had a final adult response rate ranging from $61 \%$ to $80 \% .{ }^{20}$ Most survey participants (a $94.8 \%$ average) were eligible for the mortality follow-up based on the following identifiable data combinations: (1) social security number, last name and first name; (2) social security number, sex and birthday (month, day and year); and/ or (3) last name, first name and birth month and year. ${ }^{17}$ Sampling weights adjusted for ineligible mortality linkage were used in all analyses.

Participants who responded 'yes' to the question, 'Other than during pregnancy, have you ever been told by a doctor or other health professional that you have diabetes or sugar diabetes?' were classified as having diagnosed diabetes; otherwise, participants were classified as not having been diagnosed with diabetes. The analysis sample was restricted to the 381247 adults aged $\geq 25$ years interviewed in the 1997-2011 survey years and were eligible for the mortality follow-up. Of these, 32986 reported having diagnosed diabetes and 347928 did not report having diagnosed diabetes. The analysis excluded those missing diagnosed diabetes status $(n=333)$ and persons of other race or multiple races $(n=16894)$. Therefore, the analytical sample size was 364037 (diabetes=31 586 and no diabetes $=332451$ ).

\section{Variables}

\section{Outcome}

All-cause mortality was determined by vital status after data linkage with NDI. Person-time was calculated from survey interview date to date of death or 31 December 2015 (currently the latest available mortality data) for those assumed alive. To reduce follow-up bias when assessing temporal changes in mortality rates, follow-up was right truncated at death or 5 years of follow-up (whichever came first). For example, for the 1997 cohort, mortality information was assessed up to 2001. Therefore, survey cycles after 2011 were not included since mortality rate for a 5 -year period is not available. All-cause mortality rates presented in this study are based on data of a 5-year period from the survey interview date.

\section{Socioeconomic position}

SEP was measured by two socioeconomic indicators: (1) educational attainment (completion of grades $<12$ ( $<$ high school), high school graduate or equivalency (high school grad), any education beyond high school (>high school)); and (2) the family IPR threshold (poor $<100 \%$ federal poverty level (FPL); near poor $100 \%-$ $199 \%$ FPL; middle income 200\%-399\% FPL; and high income $\geq 400 \%$ FPL). ${ }^{21}$ Education and income were self-reported. Due to missing income values of weighted percentage between 23\% and 33\% for survey years of 1997-2011, the NCHS CDC imputed missing values using reported multiple-imputation methodology producing five data sets that accompany the data release of each survey year. ${ }^{22}$ Income estimates were calculated by averaging the estimates from the five data sets and estimating the variance by calculating the within and between imputation variance. 


\section{Other covariates}

Diabetes status, age, sex and race-ethnicity were selfreported at baseline. Characteristics and demographics were described for adults with diagnosed diabetes according to three 5-year periods based on their interview date (1997-2001, 2002-2006 and 2007-2011) as counts, percentages and estimated number in the population for: sex, age groups (25-49, 50-64, 65-79 and $\geq 80$ years), race/ethnicity (non-Hispanic white, non-Hispanic black and Hispanic), educational attainment categories, IPR categories and diagnosed diabetes status. The counts for IPR were based on the imputed data set number 5 . Participants who identified as 'other race/ethnicity' were excluded from race/ethnic-specific estimates due to the limited sample size. Characteristics and demographics were also described separately for adults without diagnosed diabetes (online supplemental table 1).

\section{Statistical analysis}

Weighted Poisson regression accounting for survey design was used to calculate population-level mortality rates, weighted death number divided by the total weighted person-time and adjusted for baseline age and compared across the three survey time periods (1997-2001, 20022006 and 2007-2011). The mortality-linked adult personlevel sample weights were used in the analyses to calculate US population-level estimates. Estimates were expressed as deaths per 1000 person-years. Data were analysed for all adults and by each race/ethnic subgroup using regression model containing a three-way term for interaction between baseline diagnosed diabetes status $\times$ time period $\times$ SEP variable, including all lower order interactions and variables, and baseline age covariate . This modelling allowed comparison test between time periods, SEP levels and diabetes status. Predictive margins were used to estimate adjusted mortality rates by SEP, time period and diabetes status. Marginal effects were used to estimate strata-specific age-adjusted mortality rate differences (excess mortality risk) between those with and those without diagnosed diabetes and change in mortality rates between cohorts (1997-2001 vs 2002-2006, 2002-2006 vs 2007-2011 and 1997-2001 vs 2007-2001). Both predictive margins and marginal effects test differences using t-test.

SEP disparity was examined by defining educational attainment and IPR groups ordered from the highest to the lowest ranks. ${ }^{23} \mathrm{~A}$ ridit score for each period was calculated for education attainment and for IPR based on the midpoint of the cumulative proportion of each rank from highest to lowest, ranging between 0 (highest) and 1 (lowest). The ridit score estimates the relative position of each socioeconomic group in the social hierarchy considering their group size. ${ }^{25}$ For all adults and by each race/ ethnic subgroup, we used a Poisson regression model containing a three-way term for interaction between baseline diagnosed diabetes status $\times$ time periods $\times$ SEP ridit score and the covariate of baseline age. Absolute difference was obtained by fitting a straight line to the mortality rates ordered from the ridit score of educational attainment or IPR. The linear regression ridit slope, or SII, was interpreted as the average absolute difference in the age-adjusted all-cause mortality rate from each SEP indicator lowest to the highest rank. Relative difference, relative index of inequality (RII) expressed as a per cent change, was obtained by dividing the absolute difference by the age-adjusted all-cause mortality rate for the total population. It is interpreted as the average percentage change in the age-adjusted mortality rate from the lowest to the highest rank of each SEP indicator. From the regression model, SII and RII for each time period and diabetes status subgroup was calculated. We assessed SII and RII magnitudes for the three time periods (1997-2001, 20022006 and 2007-2011) and direction of change over time as the simple differences between the time periods $\left(1^{\text {st }}\right.$ and $2^{\text {nd }}, 2^{\text {nd }}$ and $3^{\text {rd }}$ and $1^{\text {st }}$ and $3^{\text {rd }}$ ).

For adults with no diagnosed diabetes, the results for age-adjusted mortality, SII and RII are provided in the supplemental materials (online supplemental tables 2-4). We used Stata V.15.0 in all analyses to take account of the complex multistage sampling design and to provide representative population estimates with 95\% CIs. Estimates for change from Poisson regression models were considered significant if $95 \%$ CIs did not include the null value. Since different Poisson regression models were used for each race/ethnic subgroup, comparing estimates between race/ethnic subgroups were conservatively considered statistically significantly different if $95 \%$ CIs did not overlap. ${ }^{26}$ Although we understand that this approach is very conservative, it was a better option than fitting a four-way interaction in these models which could lead to unstable or uninterpretable results.

\section{Patient and public involvement}

Patients and/or the public were not involved in the design, or conduct, or reporting, or dissemination plans of this research.

\section{RESULTS}

Between 1997-2001 and 2007-2011, the population of US adults aged $\geq 25$ years with diagnosed diabetes grew from 10.2 million to 18 million, mean age stayed steady at 60.2 years and 60.4 years and the percentage in minority racial/ethnic group (non-Hispanic black and Hispanic) increased from $29 \%$ to $31.3 \%$ (table 1 ). The percentage that had not completed high school fell from $33.3 \%$ to $25.2 \%$, and percentage living below the FPL fell from $15.5 \%$ to $14.3 \%$.

\section{Trends in all-cause mortality rates by SEP}

Among all adults with diagnosed diabetes, age-adjusted all-cause mortality rates steadily declined by $14 \%$ from 1997-2001 to 2002-2006 and 10\% from 2002-2006 to 2007-2011, a net decline from 23.5/1000 person-years (p-y) in 1997-2001 to $18.1 / 1000$ p-y in 2007-2011 or $5.4 / 1000$ p-y (table 2). In each 5-year period, all-cause mortality rates in adults with diagnosed diabetes were 


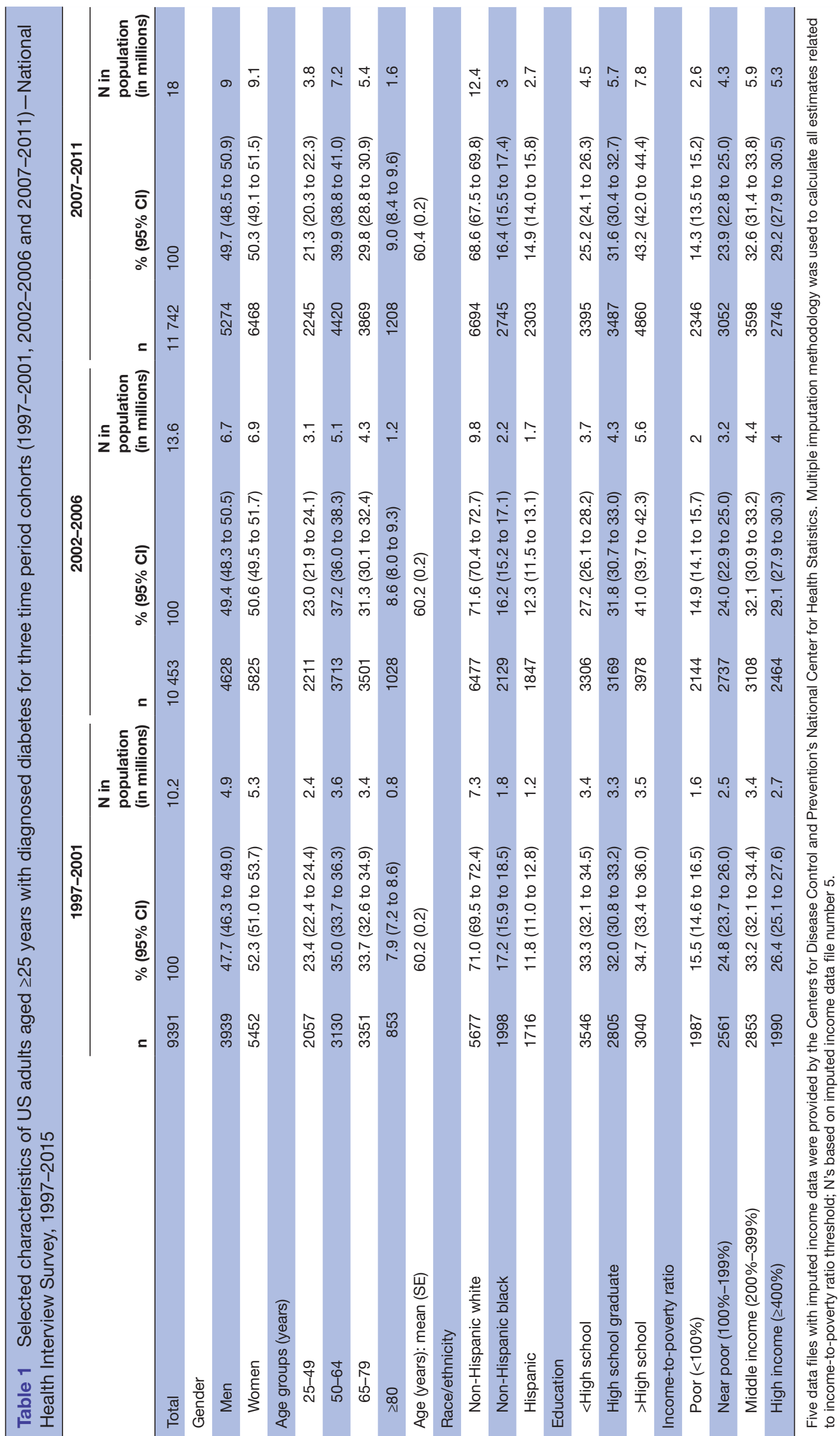




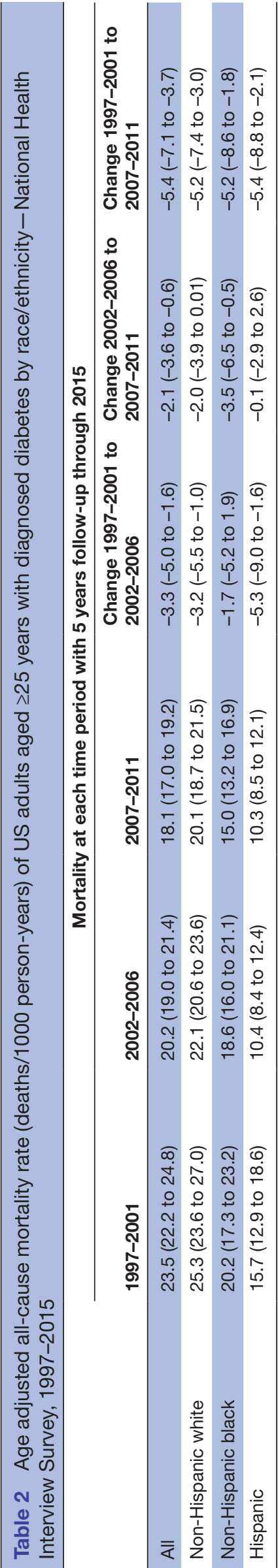

highest among non-Hispanic whites (20.1-25.3 p-y), lowest among Hispanics (10.3-15.7 p-y) and intermediate for non-Hispanic blacks (15.0-20.2 p-y) based on nonoverlapping CIs. Within each racial/ethnic group, allcause mortality rates also declined between 1997-2001, 2002-2006 and 2007-2011, but in different patterns. In non-Hispanic white adults with diagnosed diabetes, there was steady decline in mortality rates by $13 \%$ from 1997-2001 to 2002-2006 and 9\% from 2002-2006 to 2007-2011, 5.2/1000 p-y. In non-Hispanic blacks, there was no statistically significant change in mortality rate from 1997-2001 to 2002-2006, but a 19\% decline from 2002-2006 to 2007-2011. In Hispanics, there was a $34 \%$ decline in mortality rate from 1997-2001 to 2002-2006 and no statistically significant difference from 2002-2006 to 2007-2011.

Overall mortality rates among adults with diagnosed diabetes showed a significant decline between 1997-2001 and 2007-2011 in education attainment (<high school=-5.7/1000 p-y $(22 \%)$; high school graduate $=-4.2 / 1000$ p-y $(19 \%)$; and $>$ high school $=-4.8 / 1000$ p-y $(23 \%)$ ) (table 3$)$. However, the pattern of mortality rate decline of educational attainment varied by racial/ethnic group where rates declined for all levels in non-Hispanic whites (ranging from $-3.7 / 1000$ p-y to $-4.9 / 1000$ p-y $(15 \%-22 \%))$ but significantly only for the lowest educational attainment level in non-Hispanic black $(-5.0 / 1000$ p-y (23\%)) and Hispanic (-5.6/1000 p-y (34\%)) adults.

For all adults with diagnosed diabetes, there were IPR mortality rate declines for poor $=-7.9 / 1000$ p-y $(28 \%)$, middle income $=-4.7 / 1000$ p-y $(21 \%)$ and high income $=-6.2 / 1000$ p-y $(31 \%)$; but no statistically significant change for the near poor group (table 4). There were differences by race/ethnicity in the pattern of significant IPR mortality rate decline occurring in the high income $(-7.5 / 1000$ p-y $(33 \%))$ and middle income $(-4.5 / 1000$ p-y $(18 \%))$ groups, but not significantly in the two poorer groups, for non-Hispanic white adults. In contrast, mortality rates only declined significantly among the poor for non-Hispanic blacks $(-10.8 / 1000$ p-y $(38 \%))$ and Hispanics (-6.1/1000 p-y (37\%)), and not among the more affluent groups.

\section{Socioeconomic disparities in mortality}

Among all adults with diagnosed diabetes, age-adjusted all-cause mortality rates showed an inverse gradient with educational attainment (table 3) and IPR (table 4) with greater rates in the lower levels than the higher ones. Overall, the absolute education attainment disparity in all-cause mortality (SII) across the three time periods in the lowest level (<high school) than the highest (>high school) ranged from 5.9/1000 p-y to 7.4/1000 p-y greater mortality rate with no statistically significant change in the disparity over time (table 3 ). The relative disparity (RII) in the <high school than $>$ high school showed a $32.3 \%$ higher mortality rate in 1997-2001, and no statistically significant difference in the RII from the $42 \%$ in 2002-2006, and 33\% in 2007-2011. The overall absolute 


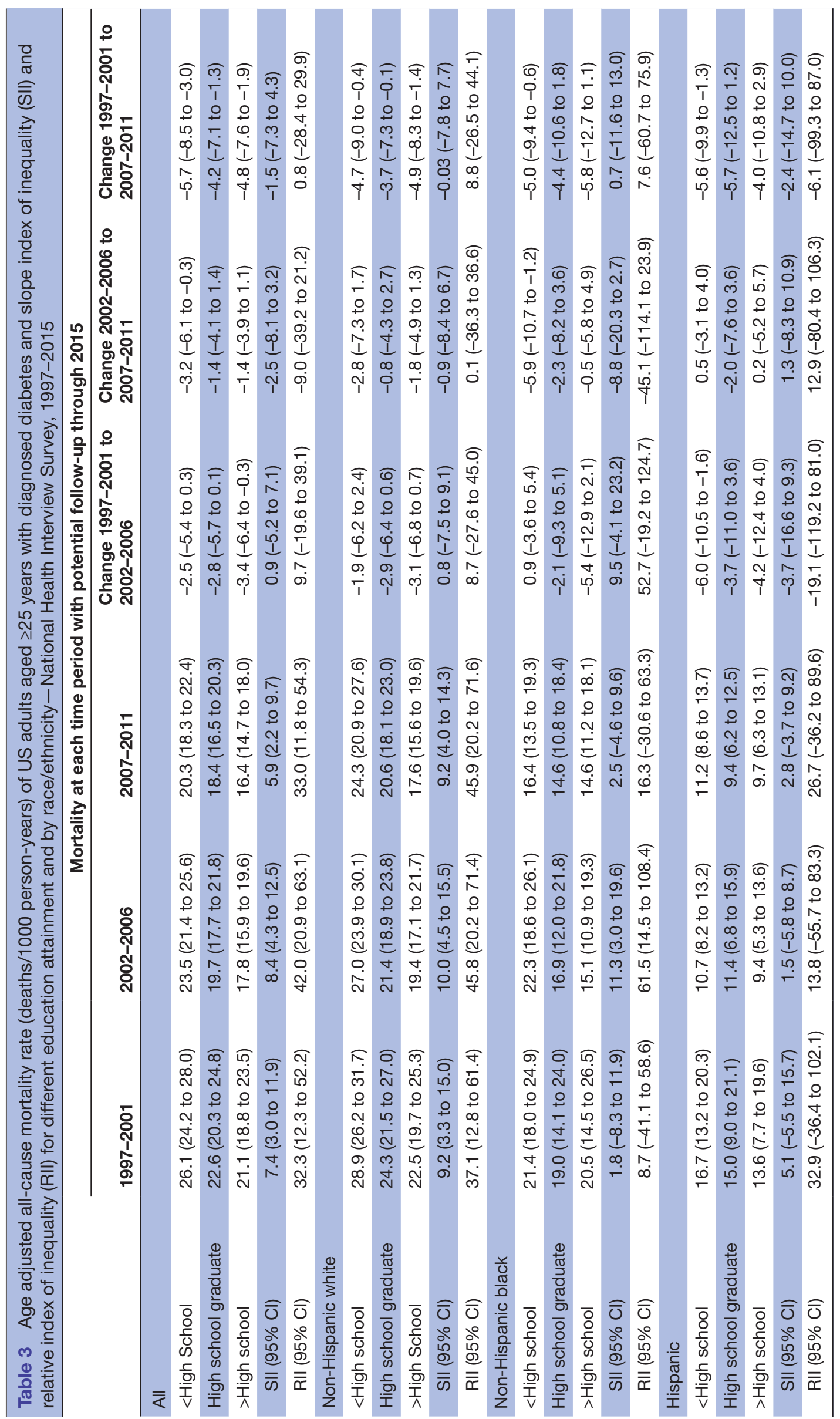




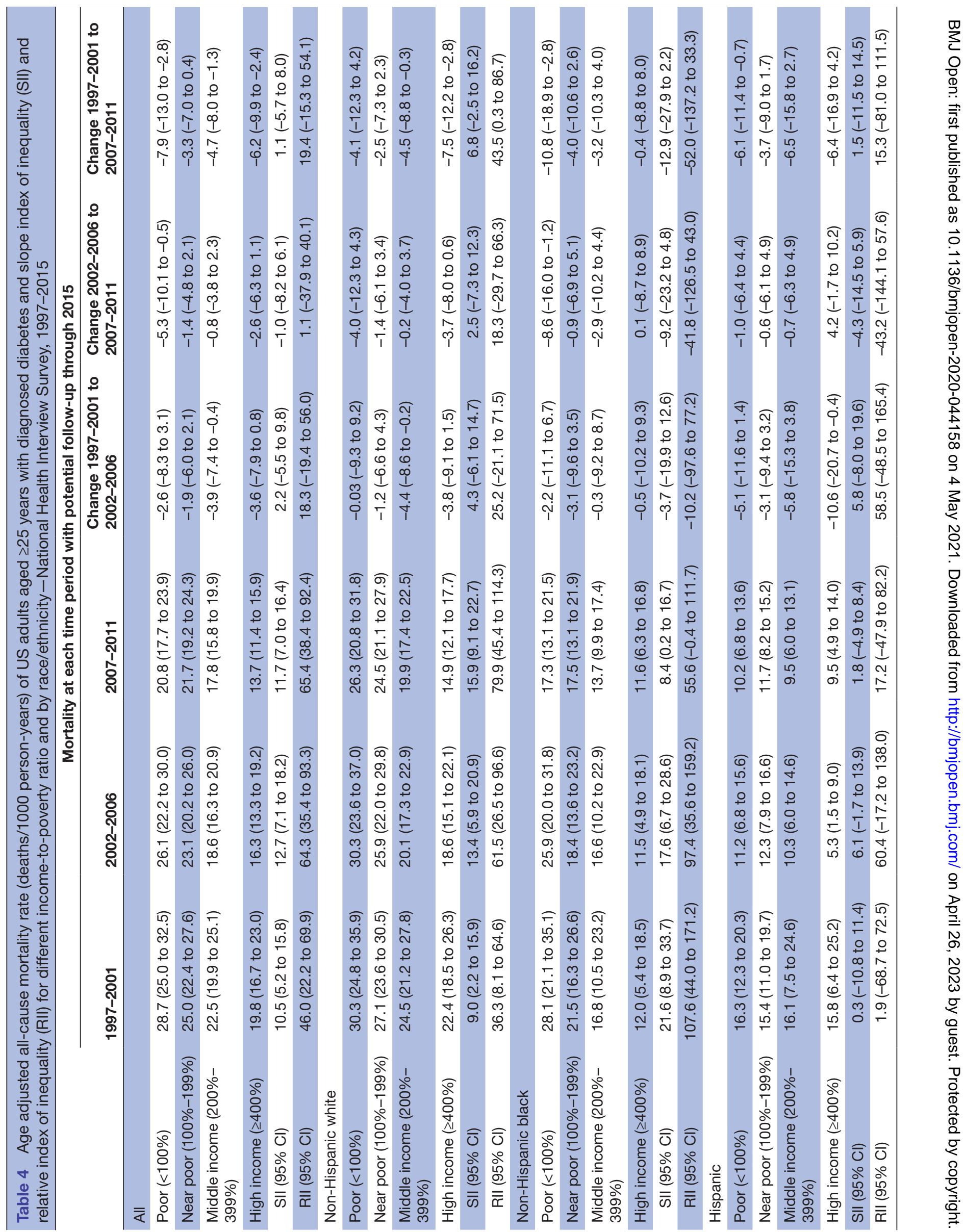


IPR disparity in all-cause mortality (SII) in the poor than the high-income group ranged from 10.5/1000 p-y to $12.7 / 1000$ p-y greater mortality rate across the three time periods, with no statistically significant change in the disparity over time (table 4 ). The relative IPR disparity (RII) in the poor than the high-income group showed a $46 \%$ higher mortality rate in $1997-2001,64.3 \%$ in $2002-$ 2006 and $64.4 \%$ in 2007-2011; but no statistical difference between the three time periods.

When comparing each strata of SEP levels and time periods across race/ethnic groups, mortality rates were mostly greater for non-Hispanic white adults than non-Hispanic black and Hispanic adults based on nonoverlapping CIs (tables 3 and 4). For non-Hispanic white adults, the absolute disparity (SII) in all-cause mortality did not significantly change across the three time periods and ranged from $9.2 / 1000$ p-y to $10.0 / 1000$ p-y for education attainment and 9.0/1000 p-y to $15.9 / 1000$ p-y for IPR. While the relative disparity (RII) for education attainment in non-Hispanic whites did not statistically significantly change and ranged from $37.1 \%$ to $45.9 \%$, the IPR RII increased from $36.3 \%$ to $79.9 \%$, a significant increase of $43.5 \%$. For non-Hispanic blacks, there was a significant education attainment disparity in all-cause mortality observed only for 2002-2006 (SII=11.3/1000 p-y and RII $=61.5 \%)$. While the IPR SII was significant across all three time periods for non-Hispanic blacks (range $=8.4 / 1000$ p-y to $21.6 / 1000$ p-y), the IPR RII was only significant in 1997-2001 (107.6\%) and in 2002-2006 $(97.4 \%)$. There were no statistically significant absolute or relative education attainment or IPR disparity in allcause mortality during these time periods for Hispanic adults with diagnosed diabetes.

\section{Excess mortality risk for adults with diagnosed diabetes}

The overall all-cause excess mortality risk in adults with diagnosed diabetes steadily decreased from 11.3/1000 p-y in 1997-2001, 8.9/1000 p-y in 2002-2006 and 7.3/1000 p-y 2007-2011, a net decrease of 4.0/1000 p-y (online supplemental table 5). The excess mortality risk tended to be greater among non-Hispanic white adults compared with non-Hispanic black and Hispanic adults. The decreased excess mortality risk for those with diabetes from 19972001 to 2007-2011 was only observed among nonHispanic white (change $=-3.8 / 1000$ p-y) and Hispanics $(-4.9 / 1000$ p-y) adults, but no significant change for nonHispanic black.

Within SEP, trends in the overall excess mortality risk for adults with diagnosed diabetes showed significant decrease at each level of educational attainment and among the poor, middle income and high income categories between 1997-2001 and 2007-2011 (figure 1). By race/ethnicity, significant excess mortality risk decreases were only observed in those with $<$ high school and $>$ high school education attainment and in the high-income group for non-Hispanic white and among the poor group in non-Hispanic black adults. Among Hispanics, there were significant decreases in excess mortality risk observed for those with < high school and high-income groups.
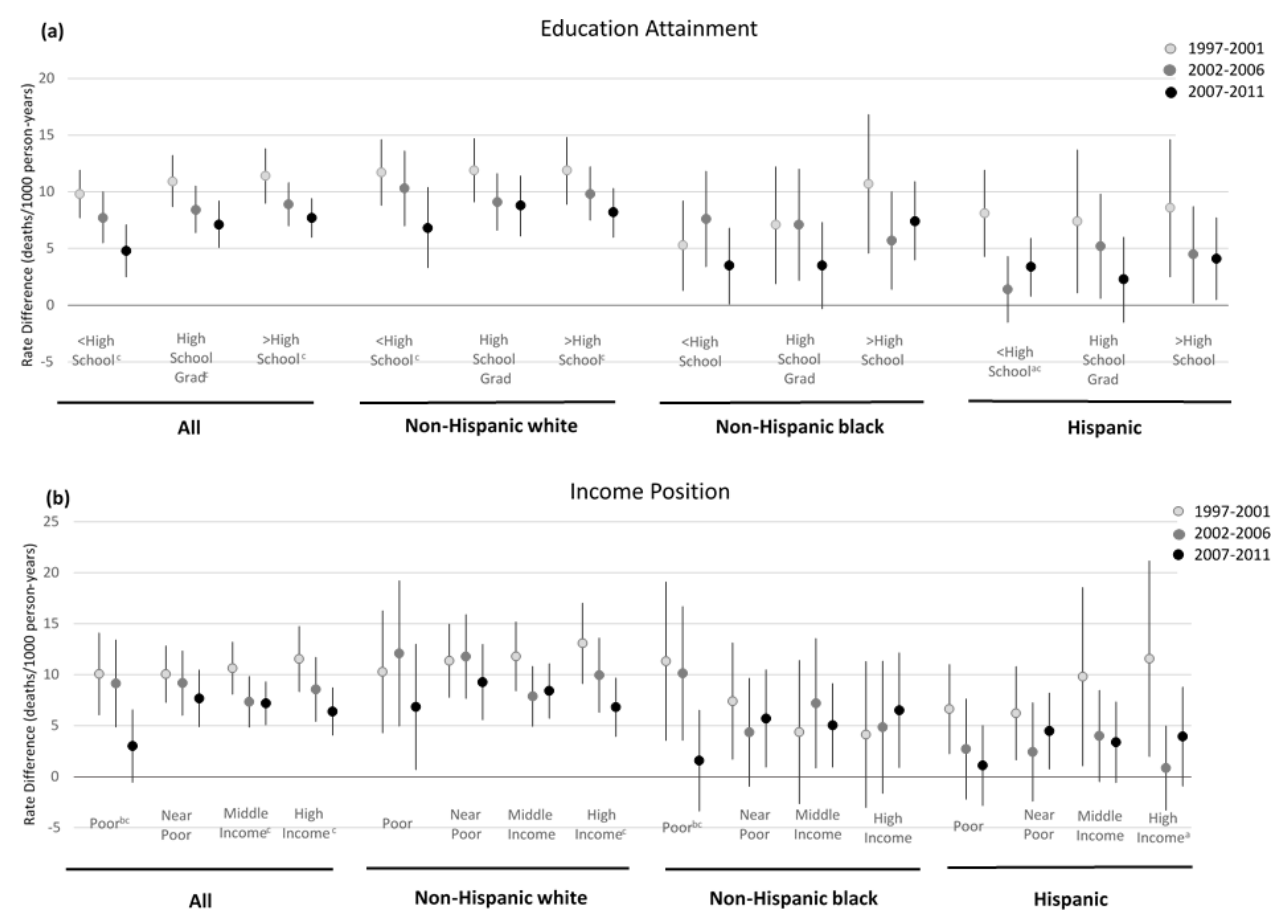

Figure 1 Age-adjusted all-cause mortality rate difference between US adults aged $\geq 25$ years with diagnosed diabetes and those without diagnosed diabetes for three time period cohorts (1997-2001, 2002-2006 and 2007-2011) - National Health Interview Survey, 1997-2015. ${ }^{\text {aP }}$ value $<0.05$ for rate difference change from 1997-2001 cohort to $2002-2006$ cohort. ${ }^{b} \mathrm{P}$ value $<0.05$ for rate difference change from $2002-2006$ cohort to $2007-2011$ cohort. ${ }^{\mathrm{C}} \mathrm{P}$ value $<0.05$ for rate difference change from 1997-2001 cohort to 2007-2011 cohort. 


\section{DISCUSSION}

In this study of a nationally representative sample of adults with diagnosed diabetes, we found age-adjusted allcause mortality rates declined from 1997 to 2011 overall and within each racial/ethnic group, and mortality rates were lower among racial/ethnic minority groups than non-Hispanic whites in each 5-year period. Age-adjusted mortality rates were inversely associated with SEP measures and significant SEP disparities in all-cause mortality rates were present overall but varied by racial/ethnic group and SEP measure. Regardless of declining mortality trends in adults with diagnosed diabetes, SEP disparity (SIIs and RIIs) did not change significantly over time meaning, for the most part, that the magnitude of the inverse association between SEP and all-cause mortality has remained constant from 1997 to 2011. The one exception was the significant increase in the IPR relative disparity (RII) of 43.5\% from 1997 to 2011 for non-Hispanic white adults with diagnosed diabetes. When considering the excess mortality risk of US adults with diagnosed diabetes, we found that the excess risk has decreased between 1997 and 2011 overall and in non-Hispanic white and Hispanic adults, but not among non-Hispanic black adults. Additionally, the excess mortality risk for adults with diagnosed diabetes has decreased within SEP level, but the changes varied throughout race/ethnic groups and SEP levels.

Our finding that age-adjusted all-cause mortality rates in adults with diagnosed diabetes were lower for nonHispanic blacks and Hispanics than the rates for nonHispanic whites is consistent with reports from studies that used US nationally representative ${ }^{12-15}$ or large convenient ${ }^{27-30}$ samples and even after adjustment for multiple covariates. This racial/ethnic patterning of allcause mortality in diabetic populations is not consistent with that of the general population's twofold greater risk reported for minority racial/ethnic groups compared with whites. ${ }^{28}{ }^{31}$ Several factors may account for these different patterns. First, in the general population the prevalence of diabetes is higher among minority racial groups than whites, so that racial/ethnic-specific mortality rates are attributable to the distribution of diabetes across the different racial/ethnic subgroups in the general population. In contrast, mortality rates in diabetic populations are estimated conditional on individuals having diabetes which removes the effect of racial/ethnic differences in prevalence. Second, despite the decline during the time period of interest, undiagnosed diabetes remained more prevalent among racial/ethnic minority groups than among non-Hispanic whites, accounting for as much as $50 \%$ of diabetes cases in racial/ethnic minority populations ${ }^{32}$; consequently, the lower rates in the diagnosed population may reflect missed undiagnosed cases and higher rates among racial/ethnic minority groups in the general population. Third, mortality rates may be lower among non-Hispanic blacks and Hispanics because they may be in better health than non-Hispanic whites at older ages when diabetes occurs. ${ }^{33}{ }^{34}$ Non-Hispanic blacks are more likely to have higher mortality rates at younger ages than non-Hispanic whites ${ }^{33}$; the high proportion of foreign-born among the current Hispanic population may contribute the assets (younger, healthier and better educated) of the 'healthy migrant'. ${ }^{34}$ In this study, we observed the age distribution of US adults with diagnosed diabetes varied by race/ethnicity with an older distribution for non-Hispanic whites (43\% aged $\geq 65$ years) compared with non-Hispanic blacks (33\% aged $\geq 65$ years) and Hispanics ( $30 \%$ aged $\geq 65$ years). Based on all these reasons, we chose to use all-cause mortality instead of disease-specific mortality to minimise bias and have a comprehensive clear outcome.

The results of this study confirm earlier reports of no excess mortality risk among racial/ethnic minority groups with diagnosed diabetes but inverse relationships between SEP measures (educational attainment, income, wealth) and mortality risk within these groups. ${ }^{12} 1315$ However, we document that within racial/ethnic groups, adults with diabetes exposed to the greatest socioeconomic disadvantage experienced significantly greater mortality burden than their more affluent peers despite declining trends in mortality. We are not aware of other evidence that SEPmortality relationships persist or worsened despite secular improvement in the health of the diabetic population, as measured by declining mortality risk. Additional analyses (online supplemental table 2 and 5) showed that among adults without diagnosed diabetes age-adjusted all-cause mortality rates were half as high as those for adults with diagnosed diabetes; yet, they experienced improvements to a much lesser degree during this period, especially by SEP measures, and SEP disparity in mortality was more consistently persistent across race/ethnic groups (online supplemental tables 3 and 4).

Although greater improvements in all-cause mortality among adults with diagnosed diabetes compared with adults without diabetes were noted, the underlying diabetic population has experienced marked changes in the distribution of the SEP indicators. For example, the proportion of adults with diagnosed diabetes reporting greater than high school education attainment increased and those with less than high school graduation decreased by 8 percentage points while only high school graduation remained at 31\% between 1997-2001 and 2007-2011. Additionally, those with IPR $\geq 400 \%$ increased by almost 3 percentage points while the proportion living below the FPL remained at about $15 \%$. Surprisingly, the population of adults without diagnosed diabetes also experienced an increase in those who reported greater than high school education attainment by about 6 percentage points but the IPR distribution remained fairly the same with a slight suggestion of an increased shift towards poorer levels. Therefore, in adults with diabetes, not only has the SEP distribution shifted towards higher SEP levels but the shape of the distribution has changed suggesting that the underlying diabetic population in 1997-2001 is not the same as the more recent 2007-2011 population. The population of adults without diagnosed diabetes seems to have experienced a different pattern in SEP distribution 
changes. Considering the inverse relationship between SEP and mortality, the distribution changes in SEP observed in the underlying populations of adults with and without diabetes can explain why greater improvements were seen in those with diabetes than those without. If health improvements are responsible for the decreasing mortality rates rather than the changes in the SEP distribution, then these improvements have not benefited adults at the highest risk (lower SEP levels) since SEP disparity gap in all-cause mortality did not significantly change during this time.

\section{Limitations an strengths}

First, diabetes status was self-reported and ascertained only at baseline. It is possible that individuals had the disease at baseline but were undiagnosed or that they developed diabetes through the course of the follow-up time. Therefore, the number of diagnosed cases may have been subject to recall and social desirability bias. However, self-reported diagnosed diabetes has been shown to have high reliability. ${ }^{32}$ These findings do not reflect disparities in the prevalence of all diabetes (diagnosed plus undiagnosed); approximately $28 \%$ of all diabetes is undiagnosed $^{32}$ and might vary by SEP as well as race/ethnicity. Additionally, we only considered self-reported diagnosed diabetes and were unable to assess diabetes management; related to quality of care, medication adherence and glycated haemoglobin levels, which may differ by SEP and race/ethnicity and associated with increased mortality. Also, to avoid bias related to the high non-response to survey questions on income, NHIS data sets with imputed income were used in all analyses. However, when using imputed data, there is the potential for misclassification. Furthermore, SEP measures (education attainment and IPR) were measured only at baseline; although education attainment did not likely change for many in this cohort of adults aged $\geq 25$ years, their income may have fluctuated. Also, SEP measures were self-reported and if income was misreported it could have a bias effect on the imputed values. In this study, we were unable to further stratify by sex or age groups due to sample size constraints. Finally, although there is potential for bias based on the exclusion of those ineligible for mortality linkage, the majority of NHIS participants were linkage eligible and we used sampling weights adjusted for ineligible linkage.

\section{CONCLUSIONS}

During the period 1997-2011, age-adjusted all-cause mortality rates improved across all levels of SEP, measured as education attainment and IPR. We observed no change or, in a few instances, worsening in the magnitude of the SEP disparities in mortality during the time period of interest across race/ethnic groups or for adults with and without diabetes. More research that investigates and identifies potential modifiable system-level factors that contribute to SEP disparity in all-cause mortality beyond diabetes and race/ethnicity is needed.
Contributors $\mathrm{CM}, \mathrm{GB}, \mathrm{YC}, \mathrm{KB}, \mathrm{SS}, \mathrm{EG}$ and $\mathrm{Gl}$ contributed to the design of this study. $\mathrm{CM}$ is the guarantor of this work and, as such, has primary responsibility over the integrity and accuracy of the data sets, data analyses, writing the paper and the final content of this project. All authors read, reviewed and approved the final manuscript.

Funding The authors have not declared a specific grant for this research from any funding agency in the public, commercial or not-for-profit sectors.

Disclaimer The findings and conclusions in this manuscript are those of the authors and do not necessarily represent the official position of the Centers for Disease Control and Prevention.

Competing interests None declared.

Patient consent for publication Not required.

Provenance and peer review Not commissioned; externally peer reviewed.

Data availability statement Data are available in a public, open access repository. NHIS data are publicly available at https://www.cdc.gov/nchs/nhis/dataquestionnaires-documentation.htm and NHIS mortality data to link is available at https://www.cdc.gov/nchs/data-linkage/mortality-public.htm.

Supplemental material This content has been supplied by the author(s). It has not been vetted by BMJ Publishing Group Limited (BMJ) and may not have been peer-reviewed. Any opinions or recommendations discussed are solely those of the author(s) and are not endorsed by BMJ. BMJ disclaims all liability and responsibility arising from any reliance placed on the content. Where the content includes any translated material, BMJ does not warrant the accuracy and reliability of the translations (including but not limited to local regulations, clinical guidelines, terminology, drug names and drug dosages), and is not responsible for any error and/or omissions arising from translation and adaptation or otherwise.

Open access This is an open access article distributed in accordance with the Creative Commons Attribution Non Commercial (CC BY-NC 4.0) license, which permits others to distribute, remix, adapt, build upon this work non-commercially, and license their derivative works on different terms, provided the original work is properly cited, appropriate credit is given, any changes made indicated, and the use is non-commercial. See: http://creativecommons.org/licenses/by-nc/4.0/.

\section{ORCID iD}

Carla Mercado http://orcid.org/0000-0002-0490-9532

\section{REFERENCES}

1 Centers for Disease Control and Prevention. National diabetes statistic report. Atlanta, GA: Centers for Disease Control and Prevention, US Department of Health and Human Services, 2020.

2 Geiss LS, Wang J, Cheng YJ, et al. Prevalence and incidence trends for diagnosed diabetes among adults aged 20 to 79 years, United States, 1980-2012. JAMA 2014;312:1218-26.

3 Selvin E, Ali MK. Declines in the incidence of diabetes in the U.S.Real progress or artifact? Diabetes Care 2017;40:1139-43.

4 Benoit SR, Hora I, Albright AL, et al. New directions in incidence and prevalence of diagnosed diabetes in the USA. BMJ Open Diabetes Res Care 2019;7:e000657.

5 Agardh E, Allebeck P, Hallqvist J, et al. Type 2 diabetes incidence and socio-economic position: a systematic review and metaanalysis. Int J Epidemiol 2011;40:804-18.

6 Maty SC, James SA, Kaplan GA. Life-course socioeconomic position and incidence of diabetes mellitus among blacks and whites: the Alameda County study, 1965-1999. Am J Public Health 2010;100:137-45.

7 Centers for Disease Control and Prevention. State, local, and national partner diabetes programs, 2019. Available: https://www.cdc.gov/ diabetes/programs/stateandlocal/index.html

8 Beckles GL, Chou C-F, Centers for Disease Control and Prevention (CDC). Diabetes - United States, 2006 and 2010. MMWR Suppl 2013;62:99-104.

9 Beckles GL, Zhu J, Moonesinghe R, et al. Diabetes - United States, 2004 and 2008. MMWR Supp/ 2011;60:90-3.

10 Beckles GL, Chou C-F. Disparities in the prevalence of diagnosed diabetes - United States, 1999-2002 and 2011-2014. MMWR Morb Mortal Wkly Rep 2016;65:1265-9.

11 Brown AF, Ettner SL, Piette J, et al. Socioeconomic position and health among persons with diabetes mellitus: a conceptual framework and review of the literature. Epidemiol Rev 2004;26:63-77.

12 Dray-Spira R, Gary-Webb TL, Brancati FL. Educational disparities in mortality among adults with diabetes in the U.S. Diabetes Care 2010;33:1200-5. 
13 Dupre ME, Silberberg M, Willis JM, et al. Education, glucose control, and mortality risks among U.S. older adults with diabetes. Diabetes Res Clin Pract 2015;107:392-9.

14 McEwen LN, Kim C, Karter AJ, et al. Risk factors for mortality among patients with diabetes: the translating research into action for diabetes (triad) study. Diabetes Care 2007;30:1736-41.

15 Saydah SH, Imperatore G, Beckles GL. Socioeconomic status and mortality: contribution of health care access and psychological distress among U.S. adults with diagnosed diabetes. Diabetes Care 2013;36:49-55.

16 Williams DR, Kontos EZ, Viswanath K, et al. Integrating multiple social statuses in health disparities research: the case of lung cancer. Health Serv Res 2012;47:1255-77.

17 Office of Analysis and Epidemiology, National Center for Health Statistics. The linkage of national center for health statistics survey data to the national death index - 2015 linked mortality file: methodology overview and analytic considerations. Hyattsville, Maryland, 2019.

18 Design and estimation for the National health interview survey, 19952004. Vital Health Stat 2 2000:1-31.

19 Parsons VL, Moriarity C, Jonas K, et al. Design and estimation for the National health interview survey, 2006-2015. Vital Health Stat 2 2014:1-53.

20 National Center for Health Statistics. Survey description, National health interview survey, 2011. Hyattsville, Maryland, 2012.

21 Krieger N, Williams DR, Moss NE. Measuring social class in US public health research: concepts, methodologies, and guidelines. Annu Rev Public Health 1997;18:341-78.

22 National Center for Health Statistics and Department of Health Information Security. Multiple imputation of family income and personal earnings in the National health interview survey: methods and examples. Hyattsville, Maryland: National Center for Health Statistics, Centers for Disease Control and Prevention, 2012.

23 Mackenbach JP, Kunst AE. Measuring the magnitude of socioeconomic inequalities in health: an overview of available measures illustrated with two examples from Europe. Soc Sci Med 1997;44:757-71.

24 Moreno-Betancur M, Latouche A, Menvielle G, et al. Relative index of inequality and slope index of inequality: a structured regression framework for estimation. Epidemiology 2015;26:518-27.

25 Irwin DJB. How to use Ridit analysis. Biometrics 1958;14:18-38.

26 Payton ME, Greenstone MH, Schenker N. Overlapping confidence intervals or standard error intervals: what do they mean in terms of statistical significance? J Insect Sci 2003;3:1-6.

27 Conway BN, May ME, Blot WJ. Mortality among low-income African Americans and whites with diabetes. Diabetes Care 2012;35:2293-9.

28 Hunt BR, Whitman S, Henry CA. Age-adjusted diabetes mortality rates vary in local communities in a metropolitan area: racial and spatial disparities and correlates. Diabetes Care 2014;37:1279-86.

29 Lynch CP, Gebregziabher M, Echols C, et al. Racial disparities in allcause mortality among veterans with type 2 diabetes. J Gen Intern Med 2010;25:1051-6.

30 Zhao W, Katzmarzyk PT, Horswell R, et al. Body mass index and the risk of all-cause mortality among patients with type 2 diabetes mellitus. Circulation 2014;130:2143-51.

31 Rosenstock S, Whitman S, West JF, et al. Racial disparities in diabetes mortality in the 50 most populous us cities. J Urban Health 2014;91:873-85

32 Menke A, Casagrande S, Geiss L, et al. Prevalence of and trends in diabetes among adults in the United States, 1988-2012. JAMA 2015;314:1021-9.

33 Olshansky SJ, Antonucci T, Berkman L, et al. Differences in life expectancy due to race and educational differences are widening, and many may not catch up. Health Aff 2012;31:1803-13.

34 Medina-Inojosa J, Jean N, Cortes-Bergoderi M, et al. The Hispanic paradox in cardiovascular disease and total mortality. Prog Cardiovasc Dis 2014;57:286-92. 